\title{
O USO DE SUBSTÂNCIAS NOCIVAS ASSOCIADAS AO COMPORTAMENTO DE RISCO DO PRATICANTE DE ATIVIDADE FÍSICA
}

Flávio Ribeiro Brandão

Gilmar Antoniassi Júnior ${ }^{4}$

\section{RESUMO}

Na sociedade moderna muitas pessoas buscam modificar a estética natural do corpo, por meio de diferentes intervenções. O uso de substância nociva tem se propagado no meio da prática em academias em busca do corpo ideal, através dos Esteróides Anabolizantes, que tem sido considerado a possibilidade mais acessível. $O$ uso indiscriminado pode desencadear diferentes reações adversas. O objetivo deste estudo consistiu em identificar o uso de substâncias nocivas associado ao comportamento de risco por praticantes de atividade física, a partir de uma revisão sistêmica de metanálise, que foi realizada em 15 artigos valendo-se de uma base de análise quantitativa. A análise realizada permitiu observar que o uso de Esteróides Anabolizantes e Andrógenos (EAA) associadas ao comportamento de risco do praticante de atividade física é corriqueiro. Verificou-se juntamente que o perfil dos usuários de EAA nos estudos analisados compreende a maioria composta por homens, estudantes universitários, idade entre 14 a 60 anos, praticantes de atividade física, motivados por estética e ganho de força. As substâncias mais apontadas nos estudos foram Deca-Durabolin Estanozolol e Decanoato de Nandrolona. Quanto à exposição ao risco à saúde prevaleceu nos estudos, à acne, a queda de cabelo, o aumento da libido e a irritabilidade. Conclui-se que o uso dos EAA's é uma realidade presente na vida de muitos praticantes de atividades físicas, fato que convida o psicólogo junto a uma equipe multidisciplinar, a realizar projetos, cujo objetivo seja disseminar informações sobre os malefícios inerentes ao uso de tais drogas, de modo propor ações preventivas e educativas junto à população exposta aos EAA.

Palavras-Chaves: Esteróides Andrógenos Anabólicos. Atividade Física. Saúde. Qualidade de vida.

\section{ABSTRACT}

The use of noxious has been spreading at the gyms by those who seek the ideal body, through the Anabolic Androgenous Steroids (AAS), which are considered the most accessible possibility. The indiscriminate use may lead to different adverse reactions. The aim of this study is identifying the use of noxious substances linked to risky behavior by those who practice physical activity, through a systemic review of the meta-analysis. The review was held

\footnotetext{
${ }^{4}$ Endereço eletrônico de contato: jrantoniassi@hotmail.com
}

Volume 1, Número 1 - Abril, 2015. 
in 15 papers, with the use of a quantitative base analysis. Through such analysis it was possible to notice that the use of AAS linked to risky behavior by those who practice physical activity is something trivial. Most of the users are men, college students, aged varying from 14 to 60 years old, practitioners of physical activity, with aesthetical and strength gain motivations. The most present substances in the studies were Deca-Durabolin, Estanozolol and Decanoato de Nandrolona. As for risks to health there were the prevalence of acne, hair loss, libido increment and irritability. One may conclude that the use of AAS is a fact in the lives of those who practice physical activity, which calls for a psychologist in a multitask team, holding projects whose aim is to spread information about the harm in using such drugs, by proposing preventive and educative actions to the population exposed to AAS.

Key words: Anabolic androgenous steroids. Physical activity. Health. Life Quality.

\section{INTRODUÇÃO}

O presente estudo deu-se por interesse pessoal devido à prática rotineira da atividade física. Neste contexto, tem-se observado que os praticantes de atividades físicas procuram programas voltados para o aumento de massa muscular e a queima de gorduras. Essa realidade reforça a percepção de que o objetivo desse perfil é buscar o culturismo ao corpo ideal. Isso, por vezes, faz com que a pessoa busque ações que os colocam em risco, como por exemplo, o uso de substâncias químicas nocivas.

Na sociedade contemporânea, o corpo vem sendo cada vez mais vislumbrado como um objeto passivo de ser modelado. Para modificar a estética natural do corpo dispõese de diferentes alternativas capazes de modelar o corpo físico. Em meio às alternativas, para atingir o objetivo, os anabolizantes podem ser vistos como uma possibilidade acessível e de baixo custo para aqueles que esperam alcançar o corpo ideal em um curto espaço de tempo. ${ }^{(1)}$

Os EAA correspondem ao composto químico de função hormonal derivado do hormônio da testosterona, que acomete o aumento da massa muscular e a redução de gordura, em razão de ter propriedades que intensificam os efeitos fisiológicos da testosterona. ${ }^{(2)}$

O uso dos EAA pode ocorrer em circunstâncias associadas à saúde, como infertilidade masculina, redução da libido; definhamento causado por doenças musculares; ausência de qualidade de vida na velhice por falta de hormônios; alguns casos de terapia de reposição hormonal para mulheres em menopausa; recuperação do sistema imunológico e da massa muscular de portadores de HIV; tratamento da obesidade para acelerar o metabolismo, dentre outros. Evidentemente, quaisquer casos mencionados acima exigem uma prescrição médica. ${ }^{(3)}$

O problema do uso indiscriminado de anabolizantes vem suscitando debates éticos, médicos, comportamental e experimental. Assim, políticas mais rigorosas e alguns Volume 1, Número 1 - Abril, 2015. 
programas educacionais prosseguem sendo importantes instrumentos para o enfretamento do uso indiscriminado de EAA para a busca do corpo ideal. ${ }^{(4)}$

Cada vez mais a prática da atividade física vem sendo recomendada por profissionais da saúde para melhorar a qualidade de vida das pessoas. Entretanto, muitos dos objetivos dos praticantes de atividade física nas academias são obtidos em longo prazo, realidade que para muitos não é satisfatória, assim, aqueles que esperam por resultados mais rápidos recorrem ao uso de anabolizantes que podem acelerar o processo para se conseguir o físico desejado.

Nessa perspectiva, o objetivo do estudo consistiu em identificar o uso de substâncias nocivas associado ao comportamento de risco por praticantes de atividade física, a partir de uma revisão sistemática baseada em uma análise qualitativa, visando buscar investigações científicas, providas de métodos pré-planejados que reunissem estudos originais com sujeitos. ${ }^{(5)}$ A fim de, identificar o perfil dos frequentadores de academias; descrever as principais substâncias utilizadas pelos praticantes de atividade física; e, analisar a exposição ao risco à saúde associado ao uso das substâncias.

A base temática da pesquisa pautou-se no uso de anabolizantes associado ao comportamento de risco do praticante de atividade física. Ao qual se utilizou os seguintes descritores: substâncias nocivas; esteróides andrógenos anabólicos; atividade física; comportamento de risco; saúde e qualidade de vida. Realizou-se a combinação, associação e cruzamento de tais termos para o levantamento do material bibliográfico. A coleta de dados da pesquisa foi realizada por meio de publicações cientificas procedente da base de dados CAPES, no período compreendido entre 1995 a 2013.

\section{ANABOLIZANTES ESTEROIDES E QUALIDADE DE VIDA}

Estudos epidemiológicos estabelecem uma relação positiva entre a prática da atividade física e a conduta saudável. Muitas pessoas buscam na atividade física um fim estético associado à qualidade de vida. Assim, a busca do corpo ideal em atividades físicas regulares, não só traz o culto ao corpo ideal a longo tempo, como oferece vários benefícios para a saúde e prevenção de doenças. ${ }^{(6)}$

A valorização simbólica do corpo tornou-se objeto de consumo na sociedade contemporânea, pois o corpo passou a ser considerado por muitos, como um bem material. A exagerada valorização da aparência corporal inscreve-se em um processo cujo corpo físico assume um papel fundamental na exteriorização da subjetividade e na construção da identidade. ${ }^{(7,8)}$

Volume 1, Número 1 - Abril, 2015. 
A subjetiva construção da identidade à imagem corporal pode ser compreendida, como a representação mental ligada vinculada a dinâmica singular do próprio corpo. O homem contemporâneo tem explorado cada vez menos suas potencialidades internas, e vem utilizando produtos tecnológicos, suplementos alimentares e drogas, como anabolizantes, em nome do conforto e da ergonomia, a fim de poupar o esforço físico. ${ }^{(9,10)}$

Estudos têm evidenciado que a prática da atividade física quando associada ao uso de EAA é motivada pelos fins estéticos e o ganho de força, devido à disposição do resultado imediato da substância. E apontam para o uso crescente dos EAA nos últimos anos, sem a prescrição ou o acompanhamento médico. ${ }^{(11)}$

Este consumo crescente evidencia a grande valorização do corpo na sociedade consumista, contemporânea e globalizada. Refletida pelas mídias sociais, a exposição da necessidade do corpo ideal padrão, o corpo sarado, cheio de músculos definidos, com barriga zerada. A exposição exagerada da mídia contribui possivelmente, para o crescente número de jovens que quer obter resultados imediatos a fim de atingir o corpo ideal, em um curto espaço de tempo. ${ }^{(12)}$

As ações influenciadoras das mídias incentiva mas pessoas a buscar o corpo ideal cultivado pela sociedade pós-moderna. A influência social em torno do corpo e o receio do julgamento de valor pelo estereótipo faz com que, as pessoas busquem a pratica da musculação associada ao resultado imediato, tornando assim evidente o risco de exposição à saúde ao qual se submetem através do uso anabolizante e as consequências que estes representam a saúde. Focando apenas no resultado instantâneo ao paralelo do corpo desejado socialmente. ${ }^{(7)}$

Fato é que anteriormente o uso de anabolizantes estava associado somente aos praticantes de competições esportivas. Porém atualmente o uso de EAA não se restringiu só a esta população, estudos tem apontado um padrão de comportamento do uso do EAA em adolescentes, jovens e adultos que estejam a fim de ganhar massa, e atingir o corpo sonhado socialmente. ${ }^{(11)}$

Evidenciando uma alarmante preocupação de ordem pública em razão dos riscos que os indivíduos se expõem ao uso destas substancias quando não está restrita a quem necessita. A situação se agrava quando uso destas substancias que estimulem a cultura do corpo ideal, tem se atingido a população jovem, demonstrando um aumento gradativo de anabolizantes, constituindo ai crescente problema de saúde pública. ${ }^{(11,7)}$

Ocorre que a prática do exercício físico quando associada ao uso de anabolizantes, e entre outras drogas, aponta para a possível exposição de danos irreversíveis à saúde em um curto tempo. Tornando assim, o que se consegue em tão pouco tempo através do uso de anabolizante, também se perde em pouco tempo. ${ }^{(6)}$

Volume 1, Número 1 - Abril, 2015. 
Estudos evidenciam que as justificativas dos usuários de anabolizantes está associado ao discurso de saúde que enaltece as consequências positivas advinda da prática da atividade física. É perceptível no estudo com usuários de EAA, que a preocupação com a saúde, manifesta na justificativa para a prática da musculação, mas não impede o uso das substâncias. Para alguns praticantes de atividades físicas, e o discurso da saúde engloba o fato da pratica da atividade física ao cuidado com o corpo a fim de favorecer ao afastamento do uso do cigarro e do álcool. ${ }^{(7)}$

Os hormônios andrógeno-anabolizantes têm seu uso difundido entre os esportistas, usualmente para aumentar a massa e potência, porém o uso é de forma ilegal. Para tanto, ocorre que nem sempre o uso de anabolizante é contra indicado, a indicação da substancia ocorre para fins terapêuticos, quando este se faz necessário em processos que visam incluir a estimulação do crescimento ósseo e muscular, apetite. Vale ressaltar que em casos crônicos e debilitantes como o câncer e a AIDS, o uso é recomendado. Estudos revelam que o uso de anabolizantes vem sendo usado por pessoas que apresentam boas condições de saúde, a fim de aumentar a massa e a potência muscular, além de melhorar a aparência física, autoestima e a capacidade de treinar em ritmo intenso. ${ }^{(3)}$

No Brasil, o consumo para fins estéticos dos anabolizantes ainda é pouco estudado ocasionando o uso em danos à saúde causados pelo consumo, mas pouco tem sido feito para prevenção do uso entre os jovens e a promoção da saúde dos jovens. ${ }^{(7)}$

Trabalhar os danos decorrentes do uso de anabolizantes é relevante conscientizar da necessidade de ater se, na relação global do contexto da prática atividade física focando em ações conjuntas que envolva a instituição, o educador físico e o psicólogo do esporte, a fim de traçar ações que reduza o uso de anabolizantes, no âmbito das práticas da atividade física. As questões envolvendo o uso de anabolizantes decorrem do fato de que o corpo se transforma em um meio o qual a pessoa busca reconstruir o seu eu interior. O que se fortalece em uma identidade fragilizada. ${ }^{(6,13)}$

É valido ressaltar que a prática regular do exercício físico traz resultados positivos, referente ao sono e aos seus distúrbios, aos aspectos psicológicos que referem ao transtorno de humor, ansiedade e a depressão, e aos aspectos cognitivos como memória e a aprendizagem. Embora os resultados da atividade física sejam de grande valia em todos os aspectos da qualidade de vida, quando este se associa ao uso de EAA resultam em prejuízos que os torna evidente e compromete tal qualidade, afetando todos os pontos positivos favorecidos pela prática da atividade. Fragilizando os aspectos físicos, estéticos e psicológicos, favorecendo somente a cultura do corpo ideal. O corpo enaltecido pela necessidade de inserção a um padrão estético social, em função do desejo de desenvolver rapidamente massa muscular e alcançar o seu status muscular. ${ }^{(13,14)}$

Volume 1, Número 1 - Abril, 2015. 
A necessidade de se ter o corpo ideal faz com que as pessoas que praticam atividade física busquem obter resultados rápidos, através do uso de anabolizante, não se preocupando com o risco e consequências que estes representam a saúde. Cultivando o resultado do paralelo do corpo desejado socialmente, e não a pratica pela qualidade de vida. ${ }^{(7)}$

O termo qualidade de vida tem aparecido com bastante frequência, geralmente relacionado à saúde, porém, com um sentido bastante genérico. No entanto, parece haver um consenso em torno da ideia dos fatores que a influenciam, o estado de saúde, a longevidade, a satisfação no trabalho, o salário, as oportunidades de lazer, as relações familiares, a disposição, o prazer e até a espiritualidade. ${ }^{(10)}$

Os praticantes de atividade física têm altos escores de qualidade de vida, principalmente para os aspectos físicos e psicológicos. Verifica-se que dentro dos diversos motivos apontados para a prática da atividade física, e a musculação, os mais frequentes são a busca pela forma estética e o condicionamento físico. A qualidade de vida aparece em quarto lugar. Para tanto, um alto grau de satisfação com os resultados da pratica de atividade física, parecem não estar associado à motivação para a prática com a expressão qualidade de vida, e sim com as expressões estéticas que a prática da atividade física proporciona. ${ }^{(10)}$

A beleza e a força física tornam-se mercadorias altamente valorizadas na sociedade contemporânea envolvendo os aspectos estéticos, o indivíduo assume o papel de gestor do seu corpo, que deve ser administrado como um capital. A fim de proporcionar status à pessoa, pois conquista admiração e popularidade no meio social. Um dado importante é que a motivação principal para o uso de anabolizantes é imediatismo na obtenção do corpo desejado. A comparação com os colegas de academia que começaram a praticar musculação ao mesmo tempo e apresentaram rápido desenvolvimento muscular aparece como estimulo para o consumo. ${ }^{(7)}$

\section{DISCUSSÃO}

O perfil dos usuários de EAA compreende em sua maioria composta por homens, estudantes universitários, idade entre 14 a 60 anos, praticantes de atividade física, alunos específicos do curso de Educação Física, praticantes de ginástica, de exercício físico de resistência, de esportes, e, por não praticantes de atividades físicas. Motivados sempre pela estética e o ganho de força.

No Brasil, não se tem uma estimativa do uso ilícito dos EAA, mas percebe-se que o perfil dos usuários encontra-se entre a faixa etária de18 a 34 anos, sendo a grande maioria do sexo masculino. Que não se limita apenas aos sujeitos considerados atletas, mas aos

Volume 1, Número 1 - Abril, 2015. 
praticantes de qualquer atividade física recreativa e por frequentadores de academias. Uma vez que o fator motivacional incide na busca do corpo ideal, no culto a beleza e na estética e melhora seu desempenho físico. ${ }^{(15,16)}$

Dados estes que podem ser identificados no estudo realizado com praticantes de atividade física das principais academias de Erechim e Passo Fundo no Rio Grande do Sul que identificaram que, o perfil dos praticantes de atividade dar-se entre pessoas do ambos os sexos, com idade de 21 a 25 anos, sendo estudantes universitários (11). Em Salvador na Bahia, o uso de anabolizantes está cada vez mais comum, entre os 18 e 34 anos de idade, do sexo masculino independente da classe social, o que torna o uso um problema de saúde pública. $^{(13)}$

No estudo realizado com a proposta de identificar as tendências atuais nos hábitos de consumo dos usuários de EAA, evidenciou que 78,4\% dos usuários de EAA, são fisiculturistas não competitivos e não atletas. ${ }^{(17)}$ Cujo uso da substância versa em aumentar o tamanho e a força muscular, de modo a melhorar o desempenho atlético e a aparência física. O mesmo dado constata-se no estudo realizado na grande Florianópolis, cuja finalidade de uso de EAA direciona-se para os fins estéticos e do treinamento muscular intenso. ${ }^{(18)}$

O uso indiscriminado dos EAA é bastante elevado nas academias brasileiras, por aqueles que praticam alguma atividade física, principalmente na região sudeste. Para os autores deste estudo, o uso abusivo dos EAA acontece em razão da falta de informações respeitantes as possíveis contra indicações dessas substâncias, que podem repercutir em diversos efeitos adversos à saúde. ${ }^{(19)}$

Às substâncias mais apontadas em um estudo realizado no Distrito Federal por aqueles que fazem uso de EAA, são o Deca-Durabolin Estanozolol e o Decanoato de Nandrolona. O fator motivacional de uso para os entrevistados é a melhora da aparência. Tais dados foram evidenciados através de um estudo realizado com estudantes do Ensino Médio de escolas públicas e particulares daquela capital. Vale comentar que as substâncias mencionadas neste estudo estão entre as mais utilizadas no mundo. ${ }^{(20)}$

Alista dos esteroides mais utilizados no mundo, segundo o Instituto Nacional do Abuso de Drogas do Governo Americano do EUA destaca dentre os esteroides orais estão os: Anadrol (oximetolona); Oxandrin (oxandrolona); Dianabol (metandrostenolona); Winstrol (estanozolol). Dentre os esteroides injetáveis: Depo-testosterone (cipionato de testosterona); Deca-Durabolin (decanoato de nandrolona); Durabolin (fenilpropionato de nandrolona); Equipoise (undecilenato de boldenona). ${ }^{(21)}$

Em realidade, os EAA's possuem dosagens intensas de hormônios e ao fazer uso a mesmo, transporta-se pequenas quantidades na circulação sanguínea, até os tecidos-alvos, a fim de produzir uma resposta fisiológica. Tais hormônios são classificados como animais, Volume 1, Número 1 - Abril, 2015. 
proteínas e peptídeos ou esteroides. Esta resposta fisiológica ocorre devido à função hormonal dos EAA, que potencializam a "sua função anabólica, responsável pelo desenvolvimento muscular, reduzindo o efeito androgênico. ${ }^{(22,23)}$

Quanto à exposição ao risco à saúde prevaleceu nos estudos analisados à acne, a queda de cabelo, o aumento da libido e a irritabilidade.

É possível prever os efeitos adversos dos anabolizantes em homens, mulheres e adolescentes: "[...] no homem, os testículos diminuem de tamanho, a contagem de espermatozoide é reduzida, podendo ocasionar a impotência, infertilidade, calvície, desenvolvimento de mamas, dificuldades ou dor para urinar e aumento da próstata." Nas mulheres podem-se perceber efeitos, como "[...] crescimento de pelos faciais, alterações ou ausências de ciclo menstrual, aumento de clitóris, voz grossa, diminuição dos seios" Os adolescentes também são vítimas destas drogas e os efeitos podem compreender:" [...] maturação esquelética prematura, puberdade acelerada levando a um crescimento raquítico. ${ }^{(15)}$

Os danos ocasionados pelos anabolizantes danificam vários aspectos do corpo humano, podendo desencadear os efeitos no cérebro com dores de cabeça, tonturas, e alterações psíquicas estão vinculados aos distúrbios de personalidade, depressão, mania, psicose, aumento nos níveis de irritabilidade podendo causar dependência e levar ao suicídio, bem como comportamento antissocial e de paranoia. $\mathrm{Na}$ laringe provoca alteração permanente nas cordas vocais especificamente em mulheres. No fígado resulta ao aumento da produção da enzima transaminase glutâmico pirúvica, o órgão passa a trabalhar com sobrecarga podendo desenvolver cirrose, tumor e icterícia. Nos rins e aparelho urinário provoca a retenção de liquido, sobrecarrega no órgão queimação e dor no trato urinário. No sistema lipídico redução HDL e aumento do LDL. Nos ligamentos ocorre maior chance de ruptura. Nos ossos a diminuição do período de crescimento na puberdade. No sistema circulatório e imunológico o aumento do número de hemácias jovens, diminuição dos glóbulos brancos e hipertensão arterial. ${ }^{(7,23)}$

De modo geral, os EAA são substâncias quimicamente associadas ao princípio ativo da testosterona e que podem contribuir para aumentar a força e a massa muscular. $O$ uso indevido destas substâncias tem evidenciado um problema de saúde pública.(24)

Os estudos apontam que o abuso dos EAA tem aumentado expressivamente nos últimos anos, sobretudo ocasionando danos nocivos que possam comprometer os sistemas cardiovascular, hepático e neuroendócrino. ${ }^{(24)} \mathrm{O}$ uso indiscriminado e de modo não terapêutico e com finalidades de melhorar o desempenho esportivo e, maiormente, estético, tem contribuindo para o aumento da prática abusiva. ${ }^{(25)}$

Volume 1, Número 1 - Abril, 2015. 
Boa parte dos estudos revela que os EAA são adquiridos em farmácias e academias, sem receita médica. Existe uma crença de que os efeitos adversos podem ser controlados ou evitados com o uso de outros medicamentos. Essa realidade pode ser em razão da falta de conhecimento e conscientização dos benefícios e prejuízos desses produtos, conforme evidenciado no estudo com praticantes de musculação em academias de Goiânia. ${ }^{(26)}$

A falta de conhecimento e conscientização dos benefícios e prejuízos dos EAA, a informação, educação e divulgação das implicações do uso destas drogas são uma excelente alternativa para a conscientização dos danos causados pelo uso indiscriminado. A fim de discutir a busca do tão imaginado corpo perfeito. Levando a reflexão da necessidade de haver um conjunto de fatores que agrupam a genética, associada ao treinamento e alimentação apropriada, resguardando sua integridade física e metal. ${ }^{(27)}$

Os estudos analisados apontam que a maioria dessas drogas entram ilegalmente no país e, ainda podem ser encontradas nas academias e farmácias para livre comercialização. Além disso, os EAA podem ser associados a outras drogas, e alguns usuários chegam a usar produtos veterinários na base de esteroides, o que pode causar graves problemas à saúde. ${ }^{(15)}$

É preciso pensar na possibilidade de um controle mais rigoroso nas vendas de EAA. Que possibilite ações fiscalizadoras integrada com ações educativas a fim de promover o uso racional de EAA. Contudo, diferentes medidas de prevenção precisam ser mais abrangentes para reduzir o uso indevido de EAA, ao qual o usuário está exposto aos EAA e seus riscos.

Lembrando-se que: "[...] a solução não está em proibir a comercialização dessas drogas. É preciso encontrar um meio de combater o uso irresponsável e indiscriminado, feito com fins meramente estéticos". (23)

Pode-se compreender a partir dessa realidade, a importância de se propor uma equipe multidisciplinar, que reúna psicólogos, nutricionistas, profissionais de Educação Física, entre outros, para realizar ações cujo objetivo seja disseminar informações referentes às implicações do uso indiscriminado e não terapêutico dos EAA, a fim de conscientizar as pessoas a não procurarem por estas substâncias, tendo em vistas a qualidade de vida e o ganho em saúde física, psíquica e social.

\section{CONCLUSÃO}

Volume 1, Número 1 - Abril, 2015. 
Através da análise realizada neste estudo permitiu observar que o uso de substâncias nocivas é comum entre muitos dos praticantes de atividades físicas. Possibilitando identificar danos irreversíveis a saúde ocasionada devido ao uso inadequado de EAA, levando inclusive à morte.

$O$ uso de EAA quando prescrito e acompanhado por médico é benéfico em circunstancia especifica de patologias que visa aumentar o tamanho e a força muscular, melhorar o desempenho atlético ou melhorar a aparência física. Porém estes são duvidosos quando é adotado sem a orientação médica, e o diagnóstico adequado, e o uso dar-se em razão especifica do culto a beleza e o corpo ideal exposto pela mídia.

Através deste estudo possibilitou confirmar à problemática que se propôs, elucidando que apesar das substâncias nocivas trazerem repercussões negativas para as pessoas, muitos usuários ainda adotam o uso indiscriminado dos EAA. Essa realidade acaba por convidar o psicólogo a atuar profissionalmente vinculado ao esporte junto a uma equipe multidisciplinar compostas por médicos, educadores físicos, fisioterapeutas e nutricionistas, a refletirem sobre possíveis medidas preventivas norteadas para sensibilizar a população de risco sobre os perigos do uso indiscriminado da substância EAA.

Tais equipes multidisciplinares podem propor medidas preventivas, como distribuição de cartilhas educativas e palestras em instituições escolares, universidades e diferentes academias, enfim, ambientes que podem estar presentes possíveis praticantes de atividades físicas que podem fazer o uso indiscriminado de EAA. Pensa-se que tais ações podem ser importantes para tentar levar informações sobre os efeitos nocivos dos anabolizantes para a saúde das pessoas.

Conclui-se que urge a necessidade de realizar investigações mais robustas e aprofundadas sobre este contexto, para seja possível construir um olhar mais crítico sobre o tema, podendo, desta forma, atuar na demanda inerente a essa área com maior proficiência.

\section{REFERÊNCIAS}

1. KYSELOVICOVA, O.; ANTALA, B.; MICHALAK, K. O uso de esteroides anabolizantes em esportistas recreativos. Fitness \& Performance Journal, mar. Abr.; 2008; 7(2):65-68. Colégio Brasileiro de Atividade Física, Saúde e Esporte Brasil.

2. SANTOS, A F. et al. Anabolizantes: conceitos segundo praticantes de musculação em Aracaju (SE). Psicologia em Estudo, Maringá, mai./ago. 2006; 11(2): 371-380.

3. MACEDO, C.L.D.; SANTOS, R.P.; PAQUALOTTO, A.C.; COPETTE, F.R.; PEREIRA, S.M.; CASAGRANDE A.; et al. Uso de esteroides anabolizantes em praticantes de musculação e/ou fisiculturismo. Rev Bras Med Esport. 1998; 4(1):13-17.

Volume 1, Número 1 - Abril, 2015. 
4. SILVA L.S.M.F.; MOREAU R.L.M. Uso de esteroides anabólicos androgênicos por praticantes de musculação de grandes academias da cidade de São Paulo. Revista Brasileira de Ciências Farmacêuticas Brazilian Journal of Pharmaceutical Sciences. jul./set., 2003; 39(3):235.

5. SILVA, E. Como avaliar e interpretar a literatura médica. In: Drummond, JP, Silva, E, Coutinho, M. Medicina baseada em evidências. $2^{a}$ ed. São Paulo: Atheneu; 2004.p. 48-56.

6. ROCA, K.F. Motivos de Adesão à prática de Ginástica de Academia. Ver Motric. 2008; 4(3):11-16.

7. IRIART, J.A.B.; CHAVES, J.C.; ORLEANS, R.G. Culto ao corpo e uso de anabolizantes entre praticantes de musculação. Cad de Saúde Púb. 2009; 25(4):773-782.

8. KEMP, K. Corpo modificado, corpo livre? São Paulo: Paulus; 2005.

9. GONÇALVES, C.O.; CAMPANA, A.N.; TAVARES, M.C. Influência da atividade física na imagem corporal: Uma revisão bibliográfica. Motri. 2012; 8(2):70-82.

10. SIMÕES, C.S.M.; SAMULSKI, D.M.; SANTIAGO, M.L.M. Analise da qualidade de vida de professores e alunos de musculação: um estudo comparativo. Rev Bras Ativ Fis \& Saud,. 2011; 16(12):107-112.

11. FRIZON, F.; MACEDO, S.M.D.; YONAMINE, M. Uso de esteroides andrógenos anabólicos por praticantes de atividade física das principais academias de Erechim e Passo Fundo/RS. Rev. Ciênc. Farm. Básica Apl. 2005. 26(3):227-232.

12. COURTINE, J.J. Os Stakhanovistas do narcisismo: Body-building e puritanismo ostentatório na cultura americana. In: Sant'anna DB. Políticas do Corpo. São Paulo: Estação Liberdade; 1995. p. 148-154.

13. IRIART, J.A.B.; ANDRADE, T.M. Musculação, uso de esteroides anabolizantes e percepção de risco entre jovens fisiculturistas de um bairro popular de Salvador, Bahia, Brasil. Cad. Saúde Pública. Rio de Janeiro. 2002; 18(5):1379-1387.

14. MELLO, M.T.; BOSCOLO, R.A.; ESTEVES, A.M.; TUFIK. S. O exercício físico e os aspectos Pscicobiologicos. Rev Bras Med Esport. 2005; 11(3):203-207.

15. Conselho Federal de Educação Física - CONFEF. Fisiculturismo. Revista E.F. set. 2002; 04.

16. MELLO, M.T.; TUFIK, S. Atividade física, exercício físico e aspectos psicológicos. Rio de Janeiro: Guanabara Koogan, 2004. 140 p.

17. PARKINSON, A.B.; EVANS, N.A. Anabolic androgenic steroids: a survey of 500 users. Department of Orthopedic Surgery, Harbor-UCLA Medical Center, Torrance, CA, USA. Journal Article. Medicine and Science in Sports and Exercise, 2006; 38(4):644-651

18. BUENO, J.P.L.; ARAÚJO, L.G. Uso de esteroides anabólicos androgênicos por praticantes de musculação da grande Florianópolis. 2010.

Volume 1, Número 1 - Abril, 2015. 
19. NOGUEIRA, F.R.S.; SOUZA, A.A.; BRITO, A.F.B. Prevalência do uso e efeitos de recursos ergogênicos por praticantes de musculação nas academias brasileiras: uma revisão sistematizada. Rev. Bras. Ativ. Fis. e Saúde. Pelotas/RS; Jan. 2013; 18(1):16-30.

20. ARAÚJO, J.P. O uso de esteroides androgênicos anabolizantes entre estudantes do Ensino Médio no Distrito Federal. Dissertação (Mestrado Educação Física) Programa de PósGraduação Strictu Sensu da Universidade Católica de Brasília, Brasília, 2003. 83f.

21. SILVA, P.R.P.; DANIELSKI, R.; CZEPIELEWSKI, M.A. Esteroides anabolizantes no esporte. Rev Bras Med Esporte, Nov/Dez 2002; 8(6).

22. CUNHA, T.S.; CUNHA, N.S.; MOURA, M.J.C.S.; MARCONDES, F.K. Esteroides anabólicos androgênicos e sua relação com a prática desportiva. Rev. Bras. Cienc. Farm. 2004; 40(2): 165-179.

23. MUNIZ, M.A.R.; COSTA, V.R. Anabolizantes: bombas-relógio nos músculos. Revista online (newsletter) maio 2009.

24. SANTOS, L.F. O uso de esteroides androgênicos anabolizantes nas academias de musculação da zona sul de Porto Velho. Monografia de Graduação. Curso de Educação Física - Universidade Federal de Rondônia. 2012. 47fl.

25. ABRAHIN, O.S.C.; SOUZA, N.S.F.; SOUSA, E.C.; MOREIRA, J.K.R.; NASCIMENTO. V.C.N. Prevalência do uso e conhecimento de esteroides anabolizantes androgênicos por estudantes e professores de educação física que atuam em academias de ginástica. Rev Bras Med Esporte. 2013; 19(1): 27-30.

26. ARAÚJO, L.R.; ANDREOLO, J.; SILVA, M.S. Utilização de suplemento alimentar e anabolizante por praticantes de musculação nas academias de Goiânia-GO, Rev. Bras. Ciên e Mov. 2002; 10(3):13-18.

27. CABRAL, A.C.; SANTOS, A.M. Esteroides anabolizantes versus perfeição corporal: quanto custa à saúde? Revista Digital - Buenos Aires. Jul. 2009; 14(134).

Volume 1, Número 1 - Abril, 2015. 